\title{
Aprendizagem e transferência de habilidades motoras no ensino de piano funcional em
} grupo

\author{
The learning and transfer of motor skills in the group \\ teaching of functional piano skills
}

Mirna Azevedo Costa* Universidade Federal do Espirito Santo (UFES), Brasil mirna.azevedocosta@gmail.com

* Mirna Azevedo Costa é pianista e professora auxiliar na Universidade Federal do Espírito Santo (UFES). Atualmente é doutoranda em Música pela Universidade Federal do Paraná (UFPR), com estágio doutoral na Université Laval (UL), no Canadá. 


\section{Resumo}

Este trabalho visa comunicar a pesquisa em andamento no campo do ensino de piano, apontando os fatores que favorecem a aprendizagem e a transferência de habilidades motoras, especialmente em contexto de ensino coletivo, com o objetivo de potencializar o aprendizado de piano como instrumento complementar no ensino superior. A metodologia utilizada é a pesquisa-ação, tendo sido realizada em dois semestres da disciplina Piano Funcional na Universidade Federal do Paraná (UFPR). Atualmente em fase de análise de dados, a pesquisa procura levantar e discutir questões relativas à aprendizagem motora e à possível utilização de estratégias de ensino visando a aprendizagem e transferência de habilidades motoras ao piano. Neste artigo, são abordados os conceitos de habilidade motora e transferência de aprendizagem motora, bem como alguns aspectos da prática que podem favorecer a aprendizagem e transferência motora: estabelecimento de metas, foco de atenção, referência pré-prática, prática randômica e prática em bloco e aprendizagem colaborativa. Por fim, apresenta-se a situação atual da pesquisa e seu desenvolvimento.

Palavras-chave: Ensino de piano. Piano em grupo. Habilidades funcionais. Aprendizagem motora.

\section{Abstract}

This paper aims to present research in progress in the field of piano teaching, pointing out the factors that favor the learning and transfer of motor skills, especially in the context of collective teaching, with the objective of enhancing piano learning as a complementary instrument in higher education. The methodology used is action research; it was carried out over two semesters of the Functional Piano discipline at the Federal University of Paraná (UFPR, Brazil). Currently undergoing data analysis, the research seeks to raise and discuss issues related to motor learning and the possible use of teaching strategies aimed at learning and transferring motor skills to the piano. In this article, the concepts of motor skill and transfer of motor learning are discussed, as well as some aspects of the practice that can favor motor learning and transference: goal setting, attention focus, pre-practice reference, random practice and block practice, and collaborative learning. Finally, the current situation of the research and its ongoing development is presented.

Keywords: Piano teaching. Group piano. Functional skills. Motor learning. 


\title{
Introdução
}

De acordo com os currículos de programas de Graduação em Música no Brasil, a aprendizagem de habilidades funcionais ao piano é requisito obrigatório para quase todo aluno que ingressa no ensino superior de Música. Tais habilidades "são aquelas que permitem ao músico o uso do piano como uma ferramenta para aprimorar outros tipos de aprendizagem musical" (YOUNG, 2013a, p.60, tradução nossa) ${ }^{1}$, como a leitura à primeira vista, harmonização, transposição etc. Estas habilidades são trabalhadas nos cursos de licenciatura e bacharelado (composição, regência e instrumentos melódicos) na disciplina Piano Funcional, "Piano Complementar", "Piano Suplementar", "Piano B" ou "Teclado", variando a denominação e o programa segundo a instituição.

Christensen (2000) observa que os estudantes raramente transferem as habilidades empregadas na prática musical de seus próprios instrumentos para a prática do piano. Além da dificuldade natural de aprendizagem de um novo instrumento, os estudantes são requisitados a desenvolver habilidades motoras complexas em um tempo relativamente curto, geralmente entre dois e quatro semestres. Como ressalta Pike (2014, p.81, tradução nossa):

\begin{abstract}
Tocar piano é uma atividade cognitiva complexa. Estudantes de música que não têm desenvolvido conhecimentos nessa área podem experimentar sobrecarga em qualquer estágio do processo de aprendizagem a partir da percepção de duas claves musicais que devem ser lidas simultaneamente, automatizando e executando as habilidades motoras necessárias para realizar uma determinada tarefa competentemente ao piano. ${ }^{2}$
\end{abstract}

Em seu estudo sobre a importância das habilidades funcionais pianísticas, Margaret Young constata que muitos músicos, "independentemente de sua carreira, relatam que teriam usado o piano mais frequentemente se fossem mais proficientes" ${ }^{\text {"YOUNG, }}$ 2013a), fato que se observa frequentemente entre alunos e ex-alunos da graduação, levando à reflexão sobre a aprendizagem dessas habilidades no ensino superior.

Segundo o levantamento realizado por Young (2013b, p.15, tradução nossa):

Embora a maioria dos professores de piano em grupo concorde que eles ensinam as habilidades necessárias para os educadores musicais, a maioria dos professores de música de escolas públicas não acredita que as classes de piano em grupo os proporcionem treinamento suficiente nas habilidades que estes usam com mais frequência. ${ }^{4}$

\footnotetext{
1 "Functional piano skills are those skills that allow a musician to use the piano as a tool to enhance other types of musical learning."

2 "Playing the piano is a complex cognitive activity. Music students who have not developed expertise in this area may experience overload at any stage in the learning process from perception of two staves of music that must be read simultaneously, automating and executing requisite motor skills, to performing a given task at the keyboard competently."

3 "[...] regardless of their career, reported that they would have used the piano more frequently if they were more proficient."

$4 \quad$ "Although most group piano instructors agree that they teach the skills needed by music educators, most public school music teachers do not believe that group piano classes provide them with sufficient training on the skills they use most frequently."
} 
Ainda que a autora se refira ao contexto de ensino norte-americano, frequentemente nos deparamos com a mesma situação ao observar os comentários de professores, alunos e ex-alunos das universidades brasileiras. Tal fato nos leva a acreditar que o contexto seja de certa forma similar, levando em consideração, inclusive, a adoção dos modelos norte-americanos de ensino de piano em grupo para a formação superior de músicos no Brasil.

Assim sendo, podemos considerar a posição de Young (2013a) ao afirmar que:

Criar um currículo de piano em grupo que efetivamente desenvolva habilidades funcionais pianísticas deveria ser uma prioridade para professores de piano em grupo e pesquisadores que trabalham na preparação de músicos profissionais. A atenção dos professores de piano em grupo deveria continuar a se concentrar no desenvolvimento de habilidades funcionais pianísticas, como leitura à primeira vista, realização de progressões de acordes, realização de escalas, leitura de grade, transposição e harmonização de melodias, visto que estas são as habilidades pianísticas que os músicos profissionais mais usam. ${ }^{5}$

Além das questões próprias da aprendizagem de habilidades funcionais pianísticas, o ensino coletivo do instrumento também apresenta inúmeros desafios decorrentes de suas particularidades metodológicas. Dentre eles, podemos citar a heterogeneidade musical e técnica dos alunos envolvidos, a escassez de material didático no Brasil que se aplique ao contexto de ensino elementar para adultos (especialmente para classes coletivas de piano) e o pouco tempo disponível para atender às necessidades individuais dos alunos em aula. Vale observar que o material nacional é escasso e quase inexistente, mas o volume de publicações estrangeiras (sobretudo americanas) é consideravelmente grande. Contudo, este material não é muito difundido no Brasil, talvez mesmo pela dificuldade de acesso e importação, especialmente no que se refere ao material para desenvolvimento de habilidades funcionais.

Dadas estas dificuldades, muitos professores de piano em grupo adotam estratégias de ensino tutorial (características da tradição de ensino individual de piano), tornando a aula coletiva uma série de curtas aulas individuais ministradas para muitos alunos com fones de ouvido. Como observa Pike (2014, p.83, tradução nossa), "muitos professores novatos de piano em grupo não aproveitam a dinâmica do grupo ou do trabalho colaborativo". ${ }^{6}$ Esta situação dificulta tanto a instrução individual, pois o aluno recebe apenas uma pequena orientação do professor a cada aula, como também a coletiva, visto que as vantagens desse formato de aulas de instrumento não são aproveitadas em sua totalidade.

Embora as classes de piano em grupo sejam uma realidade cada vez mais frequente no Brasil, ainda hoje encontramos um campo de pesquisa pouco explorado, com escassos trabalhos publicados no país sobre o assunto e pouquíssima instrução oferecida em cursos de graduação ou especialização para a formação de profissionais devidamente

\footnotetext{
$5 \quad$ "Creating a group piano curriculum that effectively develops functional piano skills should be a priority for group piano teachers and researchers working on the preparation of professional musicians. Group piano teachers' attention should continue to be concentrated on developing functional piano skills, such as sight- reading, playing chord progressions, playing scales, reading open scores, transposing melodies and harmonising melodies, as these are the piano skills that professional musicians use most."

6 "Many novice group piano teachers do not take advantage of the group dynamic or of collaborative group work."
} 
capacitados para trabalhar com ensino coletivo de instrumento. Alguns cursos de especialização em pedagogia do instrumento e pedagogia do piano têm surgido recentemente no Brasil abrangendo uma formação mais específica em relação ao ensino coletivo. Todavia, esse esforço ainda não é suficiente para garantir que tenhamos profissionais instruídos e conscientes, capazes de aplicar os princípios metodológicos adequados a cada situação de ensino, seja ela individual ou coletiva. Como resultado, a reprodução do sistema de ensino individual de piano ainda pode ser considerada uma tendência em diversas situações de aula coletiva, limitando as possibilidades de aprendizagem e restringindo os benefícios proporcionados pela metodologia de ensino coletivo.

Afora os desafios proporcionados pelo ensino coletivo, os alunos das disciplinas de piano funcional no ensino superior também dispõem de pouco tempo para a aprendizagem de habilidades funcionais complexas ao piano. Além do desafio natural de aprendizagem de um novo instrumento, especialmente tratando-se de um instrumento harmônico, os estudantes também se deparam com dificuldades de organização do estudo para que este possa ser produtivo e eficiente. Como professora desta cadeira na Universidade Federal do Espírito Santo, pude constatar que a maioria dos alunos é capaz de um bom aproveitamento na prática de seu instrumento, mas muitos deles não conseguem empregar a mesma organização de estudo para a aprendizagem das habilidades funcionais ao piano, muitas vezes resultando em um baixo aproveitamento da disciplina. Assim sendo, a utilização de estratégias de ensino que favoreçam a aprendizagem motora para esta disciplina no ensino superior poderia gerar benefícios inestimáveis para a formação destes alunos, seja no que concerne à própria formação acadêmica ou à atuação profissional. Como sugere Owen (1988, p.88, tradução nossa):

Professores de instrumento no nível de ensino superior deveriam garantir, tanto quanto possível, que os estudantes tivessem um completo entendimento de técnicas de estudo e dos benefícios de diversas formas de prática. Isso deveria incluir prática física [estudo no instrumento], prática mental, técnicas de resolução de problemas e instruções sobre o papel dos processos psicológicos na técnica de estudo. ${ }^{7}$

Portanto, frente aos desafios apresentados no ensino superior relativos às habilidades funcionais pianísticas, especialmente tratando-se da formação de músicos e professores de música, esta pesquisa pretende contribuir para o desenvolvimento da área de pedagogia do piano, buscando refletir sobre o ensino de habilidades funcionais no instrumento. $O$ objetivo é examinar as estratégias de ensino que possibilitam otimizar a aprendizagem das habilidades motoras necessárias para realização das atividades requisitadas, para que o aluno possa se valer de tais habilidades tanto em seu estudo acadêmico quanto em sua carreira profissional.

Embora as pesquisas relacionadas às diversas facetas da aprendizagem coletiva de piano e aprendizagem motora aplicada à pratica musical tenham crescido nas últimas

$7 \quad$ "Teachers of applied music at the college level should do as much as possible to insure that students have a thorough understanding of practice techniques, and the benefits of various forms of practice. These should include physical practice, mental practice, problem-solving techniques, and instruction in the role of the psychological processes in practice technique." 
décadas, a ressalva de Owen a respeito da necessidade de aprofundamento sobre o assunto certamente continua válida:

Mais pesquisas devem ser feitas sobre as condições do estudo e o aumento da eficiência do tempo de prática [estudo]. Enquanto numerosos estudos sobre prática têm sido feitos no campo da educação física e psicologia, muito pouco tem sido aprendido em relação à prática musical. (OWEN, 1988, p.87, tradução nossa). ${ }^{8}$

Ainda hoje são poucos os estudos que tratam especificamente da questão de aprendizagem motora na prática instrumental, especialmente em relação ao desenvolvimento de habilidades funcionais pianísticas para o ensino superior. Da mesma forma, são escassos os trabalhos que levam em consideração as condições de aprendizagem coletiva para o ensino de piano.

Segundo Owen (1988, p.84, tradução nossa):

A eficiência na prática [instrumental] pode ser aprimorada através de um método sistemático de instrução em técnicas de estudo. [...]. Seguindo as instruções em técnicas básicas de estudo, maior eficiência pode ser conseguida através de uma instrução sobre o papel do esquema motor na prática e na performance. ${ }^{9}$

Contudo, é importante observar que o estudo de estratégias de aprendizagem motora para o piano

[...] não diz respeito a um incalculável número de estratégias físicas que podem ser empregadas do ponto de vista do professor de piano ou pianista para se tocar melhor escalas, ou para uma execução de trilos mais rápida, e assim por diante. Em vez disso, a ênfase recai sobre uma efetiva maneira ou maneiras de se obter tais padrões de movimento, uma vez que tenham sido decididos, "automatizados" da forma mais rápida e eficiente possível. (JACOBS, 1992, p.134, tradução nossa) ${ }^{10}$

Embora o desenvolvimento técnico de habilidades funcionais mediante estratégias de aprendizagem motora seja o foco deste estudo, vale ressaltar que a presente proposta não pressupõe uma abordagem puramente tecnicista. Ao contrário, a primazia da ideia musical nos parece parte fundamental e indissociável da aprendizagem técnica, de modo que "o ato de fazer música deve ser apontado como prioridade, abordando-se a técnica como o meio para se atingirem objetivos musicais" (CHUEKE, 2006). Muitas das estratégias de aprendizagem motora partem exatamente da compreensão musical para posterior realização, como sugere Kochevitsky (1967, p.31, tradução nossa): “[...] dois aspectos - a ideia musical e os meios técnicos para realizá-la - deveriam caminhar lado a lado. A ideia

\footnotetext{
8 "More research should be done on the conditions of practicing, and increasing the efficiency of practice time. While numerous studies of practice have been done in the field of physical education and psychology, very little has been learned regarding musical practice."

$9 \quad$ "Efficiency in practice can be improved through a systematic method of instruction in practice techniques. [...] Following instruction in basic practice techniques, additional efficiency can be gained through instruction in the role of motor schema in practice and performance."

10 " [...] is not concerned with the incalculable number of physical strategies that can be employed from the piano teacher or pianist's point of view for better scale playing, faster trill execution, and so on. Rather, the emphasis will fall on an effective way or ways to get such a movement pattern, once it has been decided on, 'automaticised' as quickly and effectively as possible."
} 
musical, sempre indo ligeiramente à frente, deveria estimular o desenvolvimento técnico". ${ }^{11}$ $E$, ainda, "se a insuficiência técnica (atividade motora inadequada) pode algumas vezes ser causada por não se encontrar a posição e forma apropriada do movimento do aparato pianístico, isso é frequentemente um sintoma de pensamento musical não desenvolvido" (KOCHEVITSKY, 1967, p.45, tradução nossa).12

Diante do panorama exposto, esta pesquisa visa um aprofundamento do conhecimento da área, podendo gerar resultados positivos para o desenvolvimento da Graduação em Música, tanto no que diz respeito às classes de "Piano B", "Piano Complementar", "Piano Funcional" ou "Teclado", quanto à própria formação especializada em pedagogia do instrumento. Acredita-se que a utilização de estratégias de ensino mais eficientes do ponto de vista motor possa contribuir para um maior aproveitamento das habilidades funcionais pianísticas por parte dos alunos de graduação inseridos em tal contexto, bem como de qualquer aluno iniciante e intermediário. Espera-se que os resultados decorrentes das questões propostas possam colaborar para o desenvolvimento da pesquisa em pedagogia de piano, especialmente em relação ao ensino coletivo do instrumento, gerando proposições e reflexões aos níveis teórico e prático para a área, bem como um possível desdobramento em futuras pesquisas com o objetivo de averiguar a aplicabilidade dos princípios de aprendizagem e performance motora no estudo do piano.

\section{Habilidade motora}

Partindo do ponto de vista motor, o conceito de habilidade é definido como "capacidade de produzir um resultado de performance com o máximo grau de certeza, mínimo de energia, ou mínimo de tempo; desenvolvida como um resultado da prática" (SCHMIDT; WRISBERG, 2001, p.202). Segundo os autores, o processo de aquisição de habilidades parte da interação de três elementos básicos para que se estabeleça a experiência de aprendizagem: o aprendiz, a tarefa e o contexto, cada qual com suas variáveis a serem consideradas. O conhecimento prévio dessas condições possibilita o estabelecimento de estratégias para que a aprendizagem motora seja eficiente.

Para os autores, a experiência de aprendizagem pode ser definida como:

Mudanças nos processos internos que determinam a capacidade do indivíduo para produzir uma nova tarefa motora. Mais especificamente, restringimos nossa visão de aprendizagem de habilidades a situações nas quais as pessoas realizam tentativas deliberadas para melhorar sua performance de um particular movimento ou ação, e referimo-nos a essas situações como experiência de aprendizagem (ou o que algumas pessoas chamam de prática deliberada). (SCHMIDT; WRISBERG, 2001, p.190, grifo do autor).

\footnotetext{
11 "[...] two aspects - the musical idea and the technical means for its realization - should go hand in hand. The musical idea, always going slightly ahead, should stimulate technical development."

12 "[...] if technical insufficiency (unsuitable motor activity) can sometimes be caused by failure to find the appropriate position and movement forms of the playing apparatus, it is usually a symptom of undeveloped musical thinking."
} 
Partindo deste conceito, podemos entender a aprendizagem do piano como instrumento complementar (habilidades funcionais pianísticas) como uma experiência de aprendizagem que envolve o desenvolvimento de novas habilidades motoras. Por exemplo, a execução de um encadeamento de acordes, por mais simples que seja, exige um novo programa motor distinto daquele utilizado para executar um instrumento melódico. O programa motor pode ser entendido como um "conjunto de comandos motores que é pré-estruturado no nível executivo e que define os detalhes essenciais de uma ação habilidosa; análogo ao gerador central de padrão" (SCHMIDT; WRISBERG, 2001, p.138). E a prática, portanto, é fundamental para que esta nova habilidade motora se estabeleça como um programa motor e para que se necessite cada vez menos de uma programação consciente da resposta motora. Ou seja, para que o aluno consiga executar um encadeamento de acordes sem a necessidade de planejar conscientemente cada movimento, ele precisa praticar com o intuito de que esta nova habilidade motora se desenvolva e se estabeleça como programa motor. Dessa forma, ele seria capaz de executar melhor uma leitura com acordes, por exemplo, podendo se ater às questões musicais e expressivas da tarefa, pois já teria os movimentos devidamente internalizados a ponto de serem executados sem muito planejamento ou programação.

Com a prática, o programa motor torna-se mais elaborado, controlando séries de comportamento cada vez mais longas, e talvez até mesmo modulando várias atividades reflexas que dão suporte à meta de movimento global. Uma vez aprendidos, esses programas são armazenados na memória de longo prazo e resgatados quando necessário para preparar [...] movimentos futuros. (SCHMIDT; WRISBERG, 2001, p.140, grifo do autor).

Para os objetivos do ensino de habilidades funcionais ao piano, também se faz necessário compreender os processos de transferência de aprendizagem motora. No contexto da disciplina, o ideal seria que os alunos não apenas desenvolvessem novas habilidades motoras, mas também pudessem utilizá-las em diferentes contextos. O estudo de uma escala, por exemplo, deveria ser útil para que os alunos pudessem realizar com mais desenvoltura os dedilhados do repertório, aplicando assim a mesma habilidade motora em um contexto distinto. Portanto, a utilização de estratégias de prática do instrumento visando a transferência de aprendizagem motora pode ser útil para que o aluno tenha essas novas habilidades estabelecidas para utilizá-las tanto no decorrer das aulas como em diversas situações vindouras. Afinal, o estudo de habilidades funcionais ao piano é pensado justamente para que o aluno possa ser capaz de utilizar o instrumento em situações distintas, seja como suporte a outras disciplinas (como harmonia, composição etc.), seja em sua atuação profissional.

\section{Transferência de aprendizagem motora}

A transferência de aprendizagem no contexto motor pode ser definida como "ganho ou perda de proficiência de uma pessoa em uma tarefa como resultado da prática anterior ou experiência em outra tarefa" (SCHMIDT; WRISBERG, 2001, p.193). Trata-se 
da transferência de uma habilidade motora, ou seja, a habilidade motora adquirida pode ser novamente acessada em momentos posteriores ou "transferida" para novas situações ou contextos distintos daqueles em que a aprendizagem foi realizada. O próprio dedilhado pode ser entendido como um exemplo de transferência de aprendizagem motora, pois os padrões adquiridos em estudos de escalas serão "transferidos" para o contexto do repertório. Segundo Roskell (1996, p.1, tradução nossa), "um bom entendimento dos princípios de dedilhados de escalas e arpejos é a melhor introdução possível para aprender a dedilhar peças musicais". ${ }^{13}$ Quanto mais estes padrões estejam consolidados como um programa motor, menor será o nível de programação da resposta diante da nova tarefa, e esta, portanto, será realizada de forma mais rápida e intuitiva.

Em um estudo realizado sobre a questão do dedilhado entre pianistas, observou-se a importância da aprendizagem desses padrões escalares para a execução de tarefas pianísticas mais exigentes:

\begin{abstract}
Alguns dos participantes observaram que padrões de dedilhados que eles aprenderam nos primeiros estágios de seu desenvolvimento constituem o "substrato" em grande parte inconsciente sobre o qual muitos de seus dedilhados deliberados são sobrepostos. Sob condições de tarefas de alta demanda (como leitura à primeira vista ou o estresse da performance pública), há uma forte tendência de se voltar a estes padrões mais primitivos. O princípio pode ser expresso como a hipótese de que, sob condições de tarefas de alta demanda, mais dedilhados padronizados serão utilizados. (CLARKE et al., 1997, p.99, tradução nossa). ${ }^{14}$
\end{abstract}

Assim sendo, acredita-se que uma aprendizagem motora que possibilite a retenção e transferência de padrões de dedilhado (não apenas no que diz respeito à realização de escalas, mas também ao encadeamento de acordes) pode ser de extrema valia para os alunos em diversas situações de estudo acadêmico ou atuação profissional, pois influencia diretamente diversas outras atividades, como leitura, harmonização, transposição, improvisação etc.

A transferência de aprendizagem motora pode se dar como transferência positiva, negativa ou nula.

Quando a experiência anterior em uma tarefa inicial é benéfica à aprendizagem de uma segunda tarefa, presume-se que tenha ocorrido uma transferência positiva. Se uma experiência anterior é prejudicial ou não tem nenhuma influência, assume-se, respectivamente, uma transferência negativa ou nula. (SCHMIDT; WRISBERG, 2001, p.194, grifo do autor).

Nas aulas de piano funcional no ensino superior, as situações de transferência positiva e negativa são geralmente muito claras: frequentemente encontramos alunos que já estudaram piano e, portanto, apresentam maior facilidade com o conteúdo da

"[...] a good understanding of the principles of fingering scales and arpeggios is the best possible introduction to fingering pieces of music."

14 "A number of the participants observed that the standard fingerings which they learned at an early stage in their development constitute the largely unconscious 'bedrock' upon which much of their more deliberate fingering is superimposed. Under conditions of high task demand (such as sight reading or the stress of public performance) there is a strong tendency to revert to these more primitive patterns. The principle can be expressed as the hypothesis that under conditions of high task demand, more standard fingerings will be used." 
disciplina (transferência positiva), como também encontramos aqueles que aprenderam dedilhados e padrões pouco eficientes, muitas vezes por conta própria e sem auxílio específico para o instrumento, que acabam dificultando a realização de determinadas atividades. Um exemplo recorrente de transferência negativa é a dificuldade apresentada por estudantes de instrumentos cuja numeração para os dedos difere daquela utilizada em instrumentos de teclas, como é o caso de violonistas e instrumentistas de cordas friccionadas, que frequentemente se confundem ao realizar as atividades no piano.

Segundo Schmidt e Wrisberg (2001, p.196), "a discussão mais antiga do conceito de transferência trata da noção de 'elementos idênticos' entre tarefas", apontada por Thorndike (1914). De acordo com tal proposta, poderia haver maior transferência entre duas tarefas caso estas compartilhassem um maior número de elementos semelhantes. Essa premissa poderia embasar a possível aplicação de estudos técnicos sistemáticos, como o estudo de escalas, arpejos e padrões de encadeamento harmônico para que estas habilidades sejam consolidadas enquanto programa motor e disponibilizadas sempre que necessário, tanto em situações próximas ou similares como em situações diferentes e longínquas.

Esta poderia então ser considerada uma das finalidades do ensino do piano funcional: desenvolver programas motores que possam ser úteis em diversas situações de leitura, de harmonização, de transposição etc., para que a demanda de atenção durante a execução de tarefas complexas seja menor e, portanto, mais eficiente. Leslie Rose ressalta "a importância da aquisição de habilidades motoras para os músicos, indicando que os exercícios técnicos são o fator-chave na aquisição de respostas físicas rápidas e eficientes na performance"15 (ROSE, 2006, p.14, tradução nossa). Assim sendo, o reconhecimento e a prática de diversos padrões (escalares, rítmicos, de tonalidade, de acordes, de dedilhado etc.) podem ser trabalhados com o objetivo de que o aluno desenvolva uma resposta motora de processamento automático diante de tarefas como leitura, transposição e harmonização, permitindo que este esteja livre para concentrar-se em questões mais complexas envolvidas nas atividades acima citadas.

Alguns aspectos da prática podem ser observados e conduzidos a fim de que novos programas motores sejam desenvolvidos e se favoreça a transferência de aprendizagem. Entre eles, podemos ressaltar o estabelecimento de metas, o foco de atenção, a referência pré-prática e a estrutura da prática, como descritos a seguir.

\section{Estabelecimento de metas}

De maneira geral, os alunos são mais comprometidos com o estudo para alcançar determinados objetivos no processo de aprendizagem quando eles mesmos participam do estabelecimento das metas. Dessa forma, como observa Schmidt e Wrisberg (2001, p.191), "[os alunos] têm um melhor entendimento do objetivo de diferentes atividades de aprendizagem", o que possibilita uma compreensão mais ampla dos procedimentos 
sugeridos e pode gerar maior empenho pessoal consciente em prol da meta estabelecida, ao invés de uma simples obediência ao esquema de estudo sugerido pelo professor. Nesse sentido, destaca-se a importância do estabelecimento claro de metas e da conscientização do aluno em relação aos objetivos da disciplina e da prática, bem como em relação aos desafios motores e musicais que estes enfrentarão durante o processo. Essas metas não necessariamente precisam ser metas de performance para a execução de determinado repertório, mas podem ser também metas de processo, onde os alunos podem se ater ao próprio desenvolvimento de suas habilidades durante as aulas.

\section{Foco de atenção}

Outra variante considerada para a aprendizagem motora é a questão do foco de atenção durante a prática, que pode ser direcionado às informações internas (como o próprio movimento corporal) ou externas (como o resultado sonoro desejado).

A aprendizagem de uma dada estrutura de movimento, especialmente aquelas altamente complexas, como muitas das habilidades envolvidas na performance musical, parece se beneficiar de diferentes focos de atenção em diferentes estágios de aquisição e refinamento de habilidades. Portanto, os professores podem facilitar a aprendizagem otimizando o direcionamento da atenção do aprendiz para pontos de foco estrategicamente selecionados. (DUKE; CASH; ALLEN, 2011, p.45, tradução nossa). ${ }^{16}$

De acordo com diversas pesquisas realizadas nos campos da música e da educação física, o foco interno parece ser mais propício aos estágios iniciais de aprendizagem de movimentos complexos, bem como o foco externo parece ser mais eficiente em situações onde algum movimento relacionado à tarefa já foi automatizado (DUKE; CASH; ALLEN, 2011). Tal comportamento pode ser elucidado através da Hipótese da Ação Constrangida:

Wulf, McNevin e Shea desenvolveram o que eles denominaram de hipótese da ação constrangida para explicar os benefícios comparativos do foco externo sobre o foco interno de atenção (McNEVIN et al., 2003; WULF; McNEVIN; SHEA, 2001). De acordo com essa visão, os aprendizes que direcionam a atenção aos seus corpos começam a controlar conscientemente movimentos que há muito tempo se tornaram automatizados e requerem pouco esforço consciente. Quando o sistema motor é constrangido por este foco de atenção artificial, os processos que deveriam ocorrer automaticamente podem ser inibidos. (DUKE; CASH; ALLEN, 2011, p.47, tradução nossa). ${ }^{17}$

\footnotetext{
16 "Wulf, McNevin, and Shea developed what they termed the constrained action hypothesis to explain comparative benefits of external over internal attentional focus (McNEVIN et al., 2003; WULF; McNEVIN; SHEA, 2001). According to this view, learners who direct attention to their bodies begin to consciously control movements that have long ago become automatized and require little conscious effort. When the motor system is constrained by this contrived attentional focus, processes that would have occurred automatically may be inhibited."

17 "Wulf, McNevin, and Shea developed what they termed the constrained action hypothesis to explain comparative benefits of external over internal attentional focus (McNEVIN et al., 2003; WULF; McNEVIN; SHEA, 2001). According to this view, learners who direct attention to their bodies begin to consciously control movements that have long ago become automatized and require little conscious effort. When the motor system is constrained by this contrived attentional focus, processes that would have occurred automatically may be inhibited."
} 
De acordo com a finalidade da tarefa, o foco de atenção pode ser considerado durante a estruturação das atividades práticas de habilidades funcionais ao piano para que o estudo possa ser mais eficiente e favoreça a aprendizagem motora. Seria possível, por exemplo, pedir que os alunos pensassem no resultado sonoro desejado de uma determinada linha melódica ou encadeamento harmônico ao invés de se concentrarem na movimentação dos dedos, tirando assim o foco de atenção do movimento motor (foco interno) e colocando-o na realização musical (foco externo).

\section{Referência pré-prática}

Um importante aspecto da aprendizagem motora, que também pode ser considerado especialmente em classes coletivas de piano, é a referência pré-prática, que "envolve aprendizagem observacional, um tipo de processamento cognitivo que facilita a aprendizagem sem prática motora" (BROTZ, 2015, p.24, tradução nossa). ${ }^{18}$ Também podemos considerar essa referência pré-prática como "visualização ou audição interna" do que se deve tocar ou do que se espera tocar.

Em aulas coletivas, as instruções são frequentemente verbalizadas ou demonstradas para que todos tenham uma ideia inicial da execução da tarefa. Além desta particularidade, os alunos estão sempre observando uns aos outros, muitas vezes comparando seu próprio desempenho e buscando possíveis soluções na execução dos colegas. Essa referência pode ser utilizada de duas formas: "(1) para servir como guia para as ações do observador quando o comportamento observado deve ser reproduzido; (2) para funcionar como padrão de correção para detecção de erros entre a resposta e a representação" (JACOBS, 1992, p. 139, tradução nossa). ${ }^{19}$

Esse procedimento, especialmente em situações de instrução coletiva, pode ser aproveitado tanto para estimular a audição crítica em relação à detecção de erros, bem como para propiciar atividades de resolução de problemas, conduzindo à reflexão e à autonomia do aluno.

\section{Prática randômica e prática em bloco}

A questão da organização e condução das seções de prática instrumental também apresenta variáveis que influenciam a aprendizagem motora, podendo ser estruturada com o objetivo de se obter melhor aproveitamento para cada situação e finalidade. Podemos considerar, portanto, algumas modalidades de prática, entre as quais ressaltamos duas: a randômica e a em bloco. A prática randômica pressupõe o treinamento de diversas habilidades em atividades diferentes durante uma sessão de estudo. Já a prática em bloco pressupõe o treinamento de uma mesma habilidade de forma constante. Por exemplo: se diferentes habilidades são praticadas em uma mesma aula de piano funcional (como

\footnotetext{
18 "[...] involves observational learning, a kind of cognitive processing which facilitates learning without motor practice."

19 "(1) to serve as a guideline for the actions of the observer when the observed behaviour must be reproduced (2) to function as a standard of correctness for the detection of error between the response and the representation."
} 
encadeamento de acordes, escalas, leitura à primeira vista e harmonização de melodias), onde diversas formas de movimentos e habilidades motoras são trabalhadas, podemos considerar tal aula como uma sessão de prática randômica. Por outro lado, se apenas uma de tais habilidades é trabalhada durante toda a aula através do mesmo tipo de atividade (como o encadeamento de acordes, por exemplo), esta poderia ser considerada uma sessão de prática em bloco.

A maioria das pesquisas tem demonstrado que, quando os indivíduos praticam uma grande variedade de movimentos de forma randômica, o desempenho durante a prática é menos produtivo do que os indivíduos que praticam movimentos de uma forma em blocos. Entretanto, quando os participantes retomam o desempenho mais tarde, aqueles que originalmente praticaram sob condições randômicas demonstram retenção superior comparada à daqueles que originalmente praticaram sob condições em blocos. Portanto, nos estudos de interferência contextual, descobrimos um desses fenômenos contra-intuitivos da aprendizagem humana: desempenho inicial mais pobre (em práticas) leva a uma melhor aprendizagem! (SCHMIDT; WRISBERG, 2001, p. 247)

A pesquisa de Stambaugh (2009) demonstra resultados coerentes com os resultados de estratégias de prática randômica e em bloco descritos acima:

Como muitos outros estudos aplicados, os resultados do Experimento I foram parcialmente consistentes com a hipótese da interferência contextual: enquanto a pontuação dos grupos foi similar na fase de aquisição, o grupo randômico executou mais rapidamente na fase de retenção. [...] A vantagem de aprendizagem exibida pelo grupo randômico sugere que esta estratégia de estudo é benéfica para estudantes de clarineta iniciantes praticando pequenas tarefas técnicas, ao contrário das estratégias de estudo por repetição promovidas em muitos métodos para iniciantes, softwares de estudo e aulas. (STAMBAUGH, 2009, p.571, tradução nossa). ${ }^{20}$

Portanto, acredita-se que as mesmas estratégias práticas poderiam ser benéficas também para o estudo do piano, especialmente tratando-se das habilidades funcionais do instrumento. Assim sendo, a estruturação das sessões de prática randômica ou em bloco certamente poderia influenciar a aprendizagem motora de habilidades funcionais ao piano.

\section{Aprendizagem colaborativa}

A configuração de aulas coletivas de instrumento é sem dúvida uma excelente oportunidade para o desenvolvimento da aprendizagem colaborativa. Contudo, o fato de vários estudantes estarem aprendendo juntos em uma classe não significa necessariamente que ali aconteça a aprendizagem colaborativa. Segundo Johnson e Johnson (1994, p.32, tradução nossa), "existe uma diferença entre simplesmente ter estudantes

\footnotetext{
20 "Like many applied studies, results of Experiment I were partially consistent with the contextual interference hypothesis: although group scores were similar at acquisition, the random group performed faster at retention. [...] The learning advantage exhibited by the random group suggests this practice strategy is beneficial for beginning clarinet students practicing short technical tasks, contrary to the drill-type strategies promoted in many beginning method books, practice software, and lessons."
} 
trabalhando em um grupo e grupos estruturados de estudantes trabalhando cooperativamente"21, onde os princípios característicos da aprendizagem colaborativa podem ser trabalhados e desenvolvidos entre os participantes do grupo.

Embora a metodologia de ensino coletivo de instrumento não seja muito recente, podemos observar que muitos professores de piano ainda desconhecem os procedimentos adequados à essa modalidade de aula. Como apontado anteriormente, muitos ignoram os princípios da aprendizagem cooperativa e deixam de aproveitar as oportunidades de aprendizagem proporcionadas em tais situações. Segundo Johnson e Johnson (1994, p.11, tradução nossa):

Existem três maneiras básicas em que os estudantes podem interagir uns com os outros enquanto aprendem. Eles podem competir para ver quem é o "melhor", podem trabalhar individualmente em uma meta, sem prestar atenção aos outros estudantes, ou eles podem trabalhar cooperativamente com um grande interesse na aprendizagem de cada um tanto quanto na sua própria aprendizagem. ${ }^{22}$

Em classes coletivas de instrumento podemos encontrar qualquer uma das três situações descritas acima, dependendo fundamentalmente apenas da maneira como o professor aborda e conduz a instrução e prática dos alunos, ou seja, como o professor compreende e aplica a dinâmica de grupo em sala de aula. Em geral, a abordagem cooperativa parece ser bem mais proveitosa do que uma abordagem individual no contexto de ensino coletivo. Pike (2014) conduziu um estudo com alunos de música em situações de aprendizagem cooperativa, obtendo os seguintes resultados:

Os estudantes de educação musical que se engajaram nas 10 semanas de colaboração com seus pares demonstraram maior proficiência individual em execuções de leitura à primeira vista e harmonização. Ademais, eles demonstraram estratégias de resolução de problemas, autoeficácia aumentada, habilidade de explicar conceitos musicais básicos, empatia para com seus colegas e maior competência pianística que o grupo controle de estudantes, que concluiu o mesmo currículo. Os participantes do grupo experimental estavam mais engajados durante as aulas, se divertiram ao piano e empregaram mais estratégias de prática deliberada. Eles escolheram prioridades durante os ensaios e persistiram até que os problemas fossem solucionados. Como resultado, as notas em performance individual dos estudantes colaborativos aumentaram significativamente nos testes de leitura à primeira vista e harmonização. Resumindo, os participantes colaborativos se tornaram mais competentes em habilidades pianísticas e didáticas, que serão necessárias em sala de aula. (PIKE, 2014, p.88-89, tradução nossa). ${ }^{23}$

\footnotetext{
21 "There is a difference between simply having students work in a group and structuring groups of students to work cooperatively".

22 "There are three basic ways students can interact with each other as they learn. They can compete to see who is 'best', they can work individualistically toward a goal without paying attention to other students, or they can work cooperatively with a vested interest in each other's learning as well as their own."

23 "The music education students who engaged in 10 weeks of collaboration with peers exhibited greater proficiency in individual sight-reading and harmonization performances. Additionally, they displayed more problem-solving strategies, increased self- efficacy, the ability to explain basic musical concepts, compassion toward their peers, and more piano competency than control group students, who completed the same curriculum. Experimental group participants were more engaged during class time, enjoyed themselves at the piano, and employed more deliberate practice strategies. They set priorities during rehearsal and persisted until problems were solved. As a result, individual performance scores of collaborative students increased significantly on sight-reading and harmonization tests. Improvements in continuity, musical flow, persistence, and self-efficacy were notable. In short, the collaborative participants became more competent with the keyboard and teaching skills that they will need in the classroom."
} 
Portanto, podemos presumir que a utilização de princípios de aprendizagem cooperativa, juntamente com as estratégias de aprendizagem motoras apresentadas anteriormente, pode efetivamente influenciar na aquisição de habilidades motoras no ensino de piano em grupo, especialmente tratando-se de habilidades funcionais. A estruturação de atividades fundamentadas nesses conceitos pode tornar a aprendizagem de tais habilidades pianísticas mais significativa e efetiva para a formação de alunos e de futuros profissionais.

\section{Desenvolvimento da pesquisa}

A pesquisa foi realizada com alunos de bacharelado e licenciatura da Universidade Federal do Paraná, sendo conduzida durante dois semestres da disciplina Piano Funcional (I e II). A metodologia utilizada foi a pesquisa-ação e a estratégia de coleta de dados empregada foi a observação (participante como observador). Todas as aulas foram conduzidas pela própria pesquisadora e documentadas em um diário de campo. As avaliações realizadas durante o semestre também foram registradas em vídeo, e um questionário foi aplicado ao final de cada semestre para levantamento de informações complementares.

Atualmente em fase de análise de dados, estão sendo categorizados os temas surgidos a partir das transcrições das observações para posterior análise e discussão. Até o momento, podemos listar os seguintes temas:

- Posicionamento/movimentação das mãos (dificuldades apresentadas pelos alunos relacionadas ao posicionamento ou à movimentação das mãos durante a realização de exercícios ou repertório).

- Estratégias de ensino (estratégias utilizadas para desenvolvimento de habilidades dos alunos na realização dos exercícios propostos).

- Observações dos alunos (elementos observados pelos alunos nas análises pré-leitura).

- Dedilhado (dúvidas e dificuldades em relação ao dedilhado de exercícios e leituras).

- Foco de atenção (com relação à concentração e à fluência durante a realização de atividades).

- Automatismo motor (relação entre o visual e o motor na leitura e execução de leituras).

- Questões teóricas (dúvidas teóricas surgidas durante as aulas).

- Imprevisibilidade (dificuldades apresentadas frente às mudanças de padrões rítmicos ou melódicos já condicionados).

- Motivação (reação e feedback dos alunos em relação a determinadas atividades).

Cada uma das categorias levantadas acima será analisada e discutida a partir dos princípios de aprendizagem motora e aprendizagem coletiva, buscando uma reflexão sobre as estratégias de ensino de habilidades funcionais ao piano, especialmente no contexto do ensino superior. 


\section{Considerações finais}

O objetivo deste artigo foi essencialmente apresentar os conceitos sobre os quais vem sendo desenvolvida a pesquisa sobre a aprendizagem e transferência de habilidades motoras no ensino de piano funcional em grupo, realizada na Universidade Federal do Paraná. Partindo de uma breve contextualização das questões que instigaram o trabalho, foram apresentados os conceitos de habilidade, aprendizagem e transferência motora, bem como os elementos que lhes podem ser favoráveis em situações de ensino (tais como: estabelecimento de metas, foco de atenção, referência pré-prática, prática randômica, prática em bloco e aprendizagem colaborativa) e um breve panorama do andamento da pesquisa no presente momento.

Após categorização de todos os dados colhidos durante a execução da pesquisa, os temas surgidos serão analisados e discutidos à luz das questões de aprendizagem e transferência de habilidades motoras, com o objetivo de buscar as estratégias de ensino que possam favorecer o aprendizado de habilidades funcionais pianísticas no contexto do ensino superior de Música. Pretende-se investigar as relações entre os processos envolvidos na aprendizagem e na transferência de habilidades motoras, estes já bem documentados no campo de estudo da educação física, e as questões derivadas do contexto de ensino de habilidades funcionais pianísticas. Assim sendo, o referencial teórico apresentado será considerado para a análise de tais processos relativos às habilidades motoras no ensino de piano funcional, buscando-se refletir sobre as características e estratégias de ensino que possam levar a um melhor aproveitamento desta disciplina no ensino superior de Música. Em suma, espera-se que esta abordagem do tema possa contribuir para o desenvolvimento da pedagogia do piano, especialmente no que se refere ao ensino coletivo do instrumento enquanto ferramenta de trabalho para os alunos dos cursos de bacharelado e licenciatura.

\section{Referências:}

BROTZ, T. Dalcrozian Piano Pedagogy and Cognitive Motor Learning Theory. American Dalcroze Journal, [s. l.], v. 41, n. 1, p. 22-29, 2015.

CHUEKE, Z. Piano Funcional na Universidade: Considerações sobre Métodos e Finalidades. Revista Científica / FAP, [s. l.], v. 1, n. jan./dez., 2006. Disponível em: <http://www. fap.pr.gov.br/arquivos/File/RevistaCientifica1/ZELIA_CHUEKE.PDF>. Acesso em: 12 fev. 2017.

CLARKE, E. et al. Talking fingers: an interview study of pianists' views on fingering. Musicae Scientiae, [s. l.], v. 1, n. 1, p. 87-107, 1997. Disponível em: <http://www.uni-graz.at/ richard.parncutt/publications/CIPaRaS197.pdf>

DUKE, R. A.; CASH, C. D.; ALLEN, S. E. Focus of Attention Affects Performance of Motor Skills in Music. Journal of Research in Music Education, [s. l.], v. 59, n. 1, p. 44-55, 2011. 
JACOBS, J. P. Piano Technique As a Manifestation of Motor Control and Learning : an Investigation From the Perspectives of the Motor. 1992. Dissertação de Mestrado, University of Pretoria, [s. l.], 1992.

JOHNSON, R. T.; JOHNSON, D. W. An Overview of Cooperative Learning. In: THOUSAND, J.; VILLA, A.; NAVIN, A. (Eds.). Creativity and Collaborative Learning. Baltimore: Brookes Press, 1994.

KOCHEVITSKY, G. The Art of Piano Playing: a scientific approach. New Jersey: Summy-Birchard Inc., 1967.

CHRISTENSEN, L. A Survey of the Importance of Funcional Piano Skills as Reported by Band, Choral, Orchestra, and General Music Teachers. 2000. Tese de Doutorado, University of Oklahoma, [s. l.], 2000. Disponível em: <https://shareok.org/bitstream/ handle/11244/5993/9975796.PDF? sequence=1\&isAllowed=y>. Acesso em: 13 fev. 2017.

OWEN, J. E. Improving Instrumental Practice Techniques Through Use of a Motor Schema Theory of Learning. 1988. Tese de Doutorado, Ohio State University, [s. l.], 1988. Disponivel em: <https://etd.ohiolink.edu/!etd.send_file?accession=osu12629564318disposition=inline>. Acesso em: 15 fev. 2017.

PIKE, P. D. An Exploration of the Effect of Cognitive and Collaborative Strategies on Keyboard Skills of Music Education Students Piano Proficiency and the Undergraduate Music Education Student. Journal of Music Teacher Education, [s. l.], v. 23, n. 232, p. 79-91, 2014.

ROSE, L. P. The effects of contextual interference on the acquisition, retention, and transfer of a music motor skill among university musicians. 2006. Tese de Douotrado, Luisiana State University, [s. l.], 2006. Disponível em: <https://login.ezproxy.net.ucf.edu/ login?url=http://search.proquest.com/docview/305316758? accountid=10003\%5Cnhttp://sfx.fcla.edu/ucf?url_ver=Z39.88-20048rft_val_fmt=info:ofi/fmt:kev:mtx:dissertation\&genre=dissertations+\%26+theses\&sid=ProQ:ProQuest+Disserta>

ROSKELL, P. The Art Of Piano Fingering A New Approach To Scales And Arpeggios. London: LCM Publications, 1996.

SCHMIDT, R. A.; WRISBERG, C. A. Aprendizagem e Performance Motora: uma abordagem da aprendizagem baseada no problema. 2a ed. Porto Alegre: Artmed, 2001.

STAMBAUGH, L. A. When Repetition Isn't the Best Practice Strategy: examining differing levels of contextual interference during practice. In: (Aaron Williamon, Sharman Pretty, Ralph Buck, Eds.)INTERNATIONAL SYMPOSIUM ON PERFORMANCE SCIENCE 2009, Auckland, New Zealand. Anais... Auckland, New Zealand: European Association 
of Conservatoires (AEC), Utrecht, The Netherlands, 2009. Disponível em: <www.performancescience.org>

THORNDIKE, E. L. Educational Psychology. New York: Century, 1914.

YOUNG, M. M. University-level group piano instruction and professional musicians. Music Education Research, [s. l.], v. 15, n. 1, p. 59-73, 2013a. Disponível em: $<10.1080 / 14613808.2012 .737773 \% 5 C n h t t p: / / e z p r o x y . u n i a n d e s . e d u . c o: 8080 /$ login?url=http://search.ebscohost.com/login.aspx?direct=true\&db=a9h\&AN=85750403\&lan$\mathrm{g}=\mathrm{es} \& \mathrm{site}=\mathrm{eds}-$ live\&scope $=$ site $>$

YOUNG, M. M. Authentic Assessments in Group Piano Classes: The Effect on Performance Skills and Attitudes. MTNA e-Journal, [s. l.], v. April, p. 14-28, 2013b. Disponível em: <http://www.mtna.org/publications/mtna-e-journal/archives-2009-2013/april-2013/> 